\title{
Family size and paternal unemployment in relation to myocardial infarction
}

\author{
M. L. BURR AND P. M. SWEETNAM \\ From the MRC Epidemiology Unit, Cardiff
}

SUMMARY Men who had been in hospital for myocardial infarction (MI) were compared with other male patients in an attempt to provide evidence on the hypothesis linking MI with poverty in childhood followed by relative affluence. In each of three social class groupings MI patients came from larger families than controls, and a higher proportion of their fathers had been unemployed for more than a year during their childhood. This gives some support to the hypothesis that childhood poverty may be associated with an excess risk of MI. There was no obvious evidence of a greater improvement in social class status among the MI patients compared with the controls.

Myocardial infarction (MI) seems to be changing its social class distribution. ${ }^{1}$ During the first half of the twentieth century it was more common in Social Classes I and II, but more recently it has become commoner in Social Classes IV and V. The hypothesis has been put forward ${ }^{2}$ that poverty in childhood followed by relative affluence is a risk factor for arteriosclerotic heart disease. MI patients and controls were therefore asked about the circumstances of their childhood to see whether any obvious differences emerged consistent with this hypothesis. In particular, their fathers' occupations, their family size, and whether their fathers were unemployed were recorded as indications of possible hardship during their childhood.

\section{Methods}

The MI patients were men aged 40-69 who were among a large number of subjects routinely interviewed during their stay in several South Wales hospitals as part of a study of possible dietary factors in the aetiology of certain diseases. The controls were selected from among the other patients in the survey by the fact that they were men aged $40-69$, in the same hospitals during the same period of time, with diagnoses that were preselected as being unlikely to be related to social conditions during childhood. All the subjects were sent a short questionnaire by post, with identical covering letters, asking about their occupations, their fathers' occupations, how many brothers and sisters they had, and whether their fathers were unemployed for longer than one year during their childhood.

\section{Results}

The information was obtained from $297 \mathrm{MI}$ patients and 305 controls. The diseases for which the control patients had been admitted to hospital are shown in Table 1.

Table 1 Number of control patients in different diagnostic groups

\begin{tabular}{lc}
\hline Diagnosis & No. of patients \\
\hline Hernias & 153 \\
Benign prostatic hypertrophy & 44 \\
Varicose veins & 26 \\
Anal fistula, fissure, and abscess & 23 \\
Urinary calculus & 16 \\
Arthritis and rheumatism & 14 \\
Intestinal and peritoneal conditions & 9 \\
Haematological diseases & 7 \\
Benign tumours & 6 \\
Metabolic diseases & 4 \\
Renal disease & 3 \\
TOTAL & 305 \\
\hline
\end{tabular}

In Table 2 the cases and controls are shown in three broad social class groupings. Both MI cases and controls show a clear gradient in number of sibs, the lower social classes having the highest numbers. In each social class grouping the MI patients had more sibs than the controls. An analysis of variance showed that these two effects were independent of each other, and were both statistically significant ( $P<0.001$ for social class; $P<0.05$ for case-control difference). 
Table 2 Ages and numbers of sibs of MI cases and controls

\begin{tabular}{llll}
\hline & \multicolumn{3}{l}{ Social class of subjects } \\
\cline { 2 - 4 } & $I / I I$ & $I I I$ & $I V / V$ \\
\hline MI CASES & & 166 & 68 \\
Numbers & 63 & $55.9 \pm 0.5$ & $56.1 \pm 0.8$ \\
Age in years (mean \pm SE) & $54.0 \pm 0.9$ & $3.94 \pm 0.22$ & $4.54 \pm 0.38$ \\
No. of sibs (mean \pm SE) & $2.87 \pm 0.30$ & 3.94 & \\
CONTROLS & & & 62 \\
Numbers & 46 & 197 & $54.4 \pm 1.0$ \\
Age in years (mean \pm SE) & $55.7 \pm 1.3$ & $56.8 \pm 0.6$ & $4.00 \pm 0.34$ \\
No. of sibs (mean \pm SE) & $2.59 \pm 0.39$ & $3.34 \pm 0.18$ & 4.00 \\
\hline
\end{tabular}

Table 3 shows the social classes of the subjects' fathers arranged in the same broad groupings. There was some association between social class of fathers and sons, but both the subjects and their fathers belonged predominantly to Social Class III. Of the 293 cases and 304 controls whose fathers' occupations were known, 82 cases $(28 \%)$ and 76 controls $(25 \%)$ had risen in social class, and 64 cases (22\%) and 63 controls $(21 \%)$ had fallen, in comparison with their fathers. These differences were not statistically significant. The Table also shows the number of subjects whose fathers were unemployed for more than a year (or had died) during their childhood. In each social class grouping there is a marked difference between cases and controls in this regard, the MI patients having the higher proportion in each case. A multiple logistic regression of percentage of fathers unemployed on disease grouping and current social class showed that the association between percentage of fathers unemployed and disease grouping was statistically significant $(P<0.05)$. There was no association between percentage unemployed and current social class.

Table 3 Fathers' social class and history of unemployment for MI cases and controls

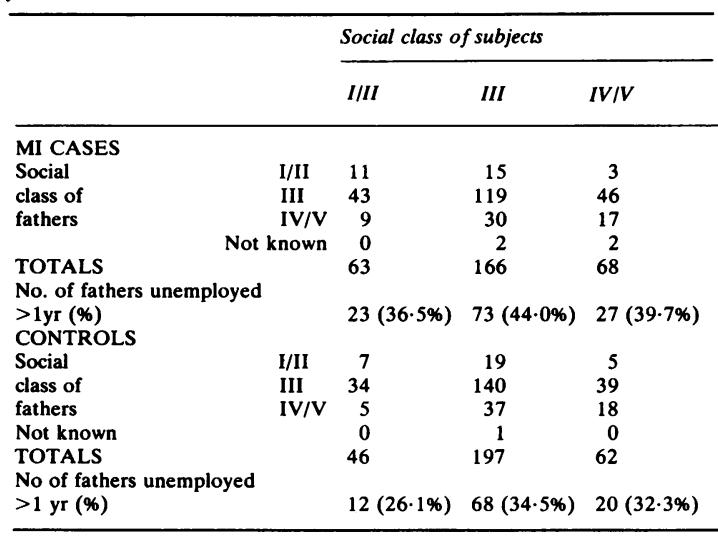

\section{Discussion}

Forsdahl ${ }^{2}$ has put forward the hypothesis that poor living conditions in childhood, followed by an improved standard of living, are an important risk factor for arteriosclerotic heart disease. He suggests that this effect may be due to changes in nutritional $\underset{\mathcal{D}}{T}$ status, so that people who have grown up in poverty have a reduced tolerance to fats which is important if ? their fat consumption rises. ${ }^{3}$ This hypothesis has the merit of explaining the observed inconsistencies in the relationship between social class and MI, which ${ }_{0}$ was formerly a disease of the more affluent and is $\overline{\bar{c}}$ now associated with the lower social classes. ${ }^{14} \frac{\widehat{D}}{}$ Presumably the upper social classes were the first and the lower social classes the most recent to show is any effect of a general rise in living standards. $i \overrightarrow{0}$

Forsdahl's hypothesis was based on a correlation between mortality rates from arteriosclerotic heart $\vec{\omega}$ disease and infant mortality rates of about 50 years ago in the different counties of Norway, which now $\stackrel{\odot}{?}$ have very similar infant mortality rates. ${ }^{2} \mathrm{He}$ has found further support for his hypothesis by demonstrating an association between seruo cholesterol in the inhabitants aged 35-49 of towns $\frac{1}{9}$ Firmmark and infant mortality in those same town 40 years earlier. $^{3}$

A strong correlation has been demonstrate between mortality from ischaemic heart disease the counties of England and Wales and infam $\vec{\varphi}$ mortality rates in these counties during periods wher $\$$ the persons concerned were young. ${ }^{5}$ It was also shown, however, that the rank order of these counties with regard to infant mortality has stayed largely the same in the past 75 years so that mortality from ischaemic heart disease correlates equally well with $\frac{}{\varnothing}$ past and present infant mortality. It is therefore impossible, on this evidence, to decide whether previous or current conditions are likely to be more important in the causation of ischaemic heart disease.

There have been several studies of the relationship between social class mobility and the risk of $\frac{\infty}{\infty}$ ischaemic heart disease. For example, Kaplan et al. ${ }^{\circ} \stackrel{\text { ? }}{?}$ found that persons from lower social class backgrounds had. an increased risk of coronary disease if their social class rose, whereas in the upper $\delta$ social class a rise in social class was associated with a $₹$ decreased risk. Gillum and Paffenbarger ${ }^{7}$ studied a $ᄋ$ cohort of former Harvard University students and found a higher risk of fatal coronary heart disease and MI among those whose fathers had a low occupational status than among those with well-to-do $\Omega$ fathers. They reviewed the findings of other surveys $\tilde{O}$ and found a consistent pattern of increased risk $\underset{\omega}{N}$ associated with intergenerational rise in social class 0 among people from low social class backgrounds. 
This they attributed to 'stress-generating conflicts, status incongruities, and life-style changes'. On the other hand, they pointed out that there is conflicting evidence on intragenerational mobility (that is, changes in social class during a person's working life); the majority of studies show no increased risks in those whose status improves. This is more difficult to explain on the basis of psychological stress, although it fits in with Forsdahl's hypothesis.

The study reported here was devised in an attempt to provide further evidence on this issue. The control subjects were all hospital patients and were therefore not necessarily representative of the general population in every respect. The possibility must be considered that the differences between cases and controls may have been due to an association between the controls' diseases and favourable circumstances in childhood. Such an association cannot be ruled out, but it seems rather unlikely in view of the nature and variety of these diseases.

Forsdahl's hypothesis has two components: firstly, that MI is associated with poverty in childhood; and, secondly, that it is related to an improvement in standard of living. The data presented here support the first of these components. In each social class grouping the MI patients had more sibs and a higher percentage of unemployed fathers than the controls. At the time of their infancy (that is, the period preceding the second world war) there was great hardship in South Wales with a high level of unemployment. Children of unemployed men at that time were likely to have been less well fed than other children, and each family's resources were divided between the members of the family, so it is reasonable to assume that less food was available per head in the larger families. Poverty may, of course, be a health hazard in several ways but the most obvious factor which may bear on long-term health is nutritional deficiency.
The second component of Forsdahl's hypothesis does not receive support from these findings, in that there is no clear evidence of a greater rise in social class status in the MI cases than in the controls. It must be recognised, however, that there is a great preponderance in both generations of Social Class III, due to the industrial background of South Wales (all colliers are in Social Class III). There has been such a rise in living standards among all sections of the local population that the lack of a difference between cases and controls in respect of change in social class should probably not be considered as important evidence against the hypothesis.

Reprints from Dr. M. L. Burr, MRC Epidemiology Unit (South Wales), 4, Richmond Road, Cardiff.

\section{References}

${ }^{1}$ Marmot MG, Adelstein AM, Robinson N, Rose GA. Changing social-class distribution of heart disease. $\mathrm{Br}$ Med J 1978; ii: 1109-12.

${ }^{2}$ Forsdahl A. Are poor living conditions in childhood and adolescence an important risk factor for arteriosclerotic heart disease? Br J Prev Soc Med 1977; 31: 91-5.

${ }^{3}$ Forsdahl A. Living conditions in childhood and subsequent development of risk factors for arteriosclerotic heart disease. J Epidemiol Community Health 1978; 32: 34-7.

${ }^{4}$ West RR. Geographical variation in mortality from ischaemic heart disease in England and Wales. Br J Prev Soc Med 1977; 31: 245-50.

${ }^{5}$ Williams DRR, Roberts SJ, Davies TW. Deaths from ischaemic heart disease and infant mortality in England and Wales. J Epidemiol Community Health 1979; 33: 199-202.

${ }^{6}$ Kaplan BH, Cassel JC, Tyroler HA, Cornoni JC, Kleinbaum DG, Hames LG. Occupational mobility and coronary heart disease. Arch Intern Med 1971; 128: 934-42.

${ }^{7}$ Gillum RF, Paffenbarger RS. Chronic disease in former college students: Sociocultural mobility as a precursor of coronary heart disease and hypertension. Am J Epidemiol 1978; 108: 289-98. 